\title{
Dificultades para la generación de evidencia sobre la efectividad de los cuidados paliativos
}

Difficulties for evidence production about palliative care effectiveness.

Zimmermann C y col. JAMA.2008; 299 (14):1698-1709.

Objetivo

CRevisar la evidencia sobre la efectividad de los equipos especializados en cuidados paliativos (CP).

\section{Fuentes de datos y criterios de selección}

Utilizando las palabras paliativos, terminal, hospice, "calidad de vida", "calidad de la atención", satisfacción y "costo económico"; los autores consultaron las principales bases de datos bibliográficos desde su creación hasta 2008. Seleccionaron investigaciones aleatorizadas que hubieran evaluado la intervención de un servicio de CP -entendido como un equipo de profesionales que ofrece o coordina la atención integral de pacientes con enfermedad terminal (ET)- y medido calidad de vida, satisfacción con la atención o costo económico.

\section{Resultados}

La evidencia de la efectividad de los equipos de CP es escasa, no habiéndose podido realizar una síntesis cuantitativa debido a la heterogeneidad de los 22 estudios identificados, sus intervenciones y sus resultados. A pesar de que muchas de las investigaciones identificadas presentan debilidades metodológicas que limitan la calidad de sus conclusiones, existe consistente evidencia de que la intervención de equipos de CP se asocia a una mayor satisfacción del la familia y/o el cuidador del paciente con ET (siete de los diez estudios que evaluaron este aspecto).

Los estudios de medición directa de $\operatorname{costos}^{\S}$ pusieron de manifiesto que los gastos hospitalarios fueron menores cuando los pacientes fueron remitidos a un programa de $\mathrm{CP}$, aumentando los costos en el hogar y en el hospice.

\section{Debilidades metodológicas}

En varios trabajos los pacientes fueron aleatorizados después de haber sido derivados a un equipo de CP, lo que puede implicar un sesgo de selección*. Si bien la "calidad de vida" fue frecuentemente seleccionada como resultado primario para la evaluación de la eficacia de los equipos de CP, sólo uno de los estudios reportó el uso de una herramienta diseñada específicamente para la población de ET. Algunos estudios sólo evaluaron el "control de síntomas", estrategia imprescindible pero que sólo refleja un aspecto del amplio, complejo y multidimensional trabajo en $\mathrm{CP}$, con el riesgo de ser reduccionista respecto de lo que significa "calidad de vida".

A pesar de que la satisfacción de los pacientes y sus cuidadores es un indicador importante de un cuidado de calidad, su evaluación suele ser problemática por varios motivos: la variabilidad subjetiva dependiente de aspectos socioculturales en la definición de lo que es una "atención satisfactoria", la posible renuencia de los pacientes para criticar a sus proveedores de cuidado y la posibilidad de satisfacción, aún con atenciones subóptimas según los estándares establecidos.

Palabras clave: calidad de vida, cuidado paliativo, hospice.

Key words: quality of life, paliative care, hospice.

Fuente de financiamiento: National Cancer Institute of Canada y Canadian Cancer Society.

\section{Comentario}

A pesar de que se usó una adecuada definición de equipo de $\mathrm{CP}$, sólo 12 de los estudios incluidos cumplieron con este criterio de inclusión y como remarca Bruera ${ }^{1}$, al no requerirse formación o maestría en $\mathrm{CP}$, el uso dicho término puede resultar engañoso.

Considero que los CP son eficaces como respuesta a necesidades de los individuos en situación de terminalidad, pero la vivencia de esta realidad es diferente si se desarrolla en el hogar, en el hospice o en un hospital; o si se llega a ella por cáncer, síndrome de la inmunodeficiencia adquirida (SIDA), enfermedad pulmonar obstructiva crónica o enfermedades neurológicas; ya que estos subgrupos pueden tener diferentes necesidades, recursos y complejidades.

Como las mediciones de calidad de la vida basadas fundamentalmente en la situación funcional no son pertinentes para valorar el impacto del $\mathrm{CP}$, debería ponerse mayor énfasis en lo que se refiere a una comunicación efectiva, así como a la planificación y la preparación del final de la vida ${ }^{2}$, recordando que la experiencia de sufrimiento solo puede entendida por expe- riencia directa, difícilmente cuantificable y comparable ${ }^{1}$. Respecto del análisis de los costos, es de esperar que al trasladarse el lugar de atención, aumenten los gastos en esa nueva área. Por lo tanto, deberían considerarse en relación al uso racional de los recursos, analizando la utilidad, el beneficio y futilidad de los mismos.

\section{Conclusiones de la comentadora}

La evaluación de la eficacia de los CP a través de investigaciones aleatorizadas puede ser dificultosa por sesgos en el reclutamiento y dificultades en la adherencia a los protocolos;, lo que puede limitar su validez externa ${ }^{3}$. Además, podemos preguntarnos si es ético aleatorizar pacientes en fase terminal a no recibir un tratamiento que podría mejorar su calidad de vida o aliviar su sufrimiento. Por lo tanto, son necesarios ensayos bien planificados y que utilicen una normalización de las intervenciones de $\mathrm{CP}$, con estándares definidos y herramientas específicas de evaluación de los resultados de este tipo de intervenciones validadas en la población a estudiar.

Liliana Rodrguez [ Sección Cuidados Paliativos del Hospital Italiano de Buenos Aires. liliana.rodriguez@hospitalitaliano.org.ar ]

Rodríguez L. Dificultades para la generación de evidencia sobre la efectividad de los cuidados paliativos. Evid. actual. práct. ambul; 11(6):167, NovDic.2008. Comentado de: Zimmermann C y col. Effectiveness of Specialized Palliative Care: A Systematic Review. JAMA. 2008; 299(14):16981709. PMID: PMID: 18398082. Disponible en URL: http://jama.ama-assn.org/cgi/reprint/299/14/1698.

\section{Referencia}

1. Bruera. Studying the Effectiveness of Palliative Care. JAMA.2008; 300: 1022

2. Eisenchlas $\mathrm{H}$ y col. Use of the Palliative Outcome Scale in Argentina: A Cross-Cultural Adaptation

and Validation Study J. Journal of Pain and Symptom Management (en prensa, aceptado en 2007).

3. Aoun S y col. Evidence in palliative care research: how should it be gathered? MJA. 2005; 183(5): 264-266.

$\$$ Cabe aclarar que los análisis de costo-efectividad y/o utilidad medidos en función de costo por unidad de tiempo ajustada por calidad de vida pueden nos ser apropiados para evaluar programas de atención en el final de la vida, dado el corto tiempo de duración de la atención prestada y las consideraciones sociales y personales de valor de calidad. ; Por ejemplo, los médicos que permiten que sus pacientes gravemente enfermos participen de estos estudios y los pacientes dispuestos a ser incluidos pueden ser poco representativos de la población general, lo que llevaría a resultados poco generalizables, aun con estudios de buena calidad metodológica. 